\title{
Thyroid Agent
}

National Cancer Institute

\section{Source}

National Cancer Institute. Thyroid Agent. NCI Thesaurus. Code C1553.

Compounds that influence the function of the thyroid hormone through replacement, inhibition or stimulation. $(\mathrm{NCl})$ 